Abstract

\title{
Small RNAs Virome Characterization Reveals Arthropod-Associated Viruses in Anopheles atroparvus from the Ebro Delta, Spain ${ }^{\dagger}$
}

\author{
Lotty Birnberg ${ }^{1}$, Francisco M. Codoñer ${ }^{2}$, Raúl Escosa ${ }^{3}$, Carles Aranda ${ }^{1}$, Ana Isabel Núñez ${ }^{1}$, \\ Sandra Talavera ${ }^{1}$ and Núria Busquets ${ }^{1 *}$
}

1 IRTA, Centre de Recerca en Sanitat Animal (IRTA-CReSA, Campus de la Universitat Autònoma de Barcelona, 08193 Bellaterra, Spain; lotty.birnberg@irta.cat (L.B.); carles.aranda@irta.cat (C.A.); anaisabel.nunez@irta.cat (A.I.N.); sandra.talavera@irta.cat (S.T.); nuria.busquets@irta.cat (N.B.)

2 Lifesequencing, Parc Cientific Universitat de Valencia, 46980 Paterna, Spain; current address: Danone, Nutricia Research, 30th Biopolis Street, Matrix Building, 138671, Singapore; fcodoner@gmail.com

3 Consorci de Politiques Ambientals de les Terres de l'Ebre (COPATE), Plaça de Lluis Companys, 43870 Amposta, Spain; rescosa@copate.cat

* Correspondence: nuria.busquets@irta.cat

† Presented at Viruses 2020-Novel Concepts in Virology, Barcelona, Spain, 5-7 February 2020.

Published: 24 June 2020

\begin{abstract}
Even though malaria was eradicated from Europe after the mid-20th century, in 2017, more than 8000 imported cases were reported in the continent. Due to travel routes to endemic areas, climate change, and the presence of native vector mosquitoes (genus Anopheles), the reestablishment of autochthonous malaria transmission is a current concern. Anopheles atroparvus (Van Thiel, 1972) is one of the 11 sibling species within the Palearctic Anopheles maculipennis complex, which formerly were considered the main vectors of the disease in the European continent. The microbiota (bacteria and viruses) of vector species has been demonstrated to play a significant role in the biology of these organisms, including their infection susceptibility and their capacity to transmit disease-causing agents. Recently, with the improvement of metagenomics techniques, several viruses that naturally infect vector mosquitoes have been identified. The purpose of the present study was to characterize, for the first time, the virome present in An. atroparvus from the Ebro Delta and assess its evolution after ten generations in the laboratory. Small RNA sequencing was used to characterize the virome from wild-caught An. atroparvus females and from the tenth generation produced under controlled laboratory conditions. Through this approach, we were able to identify viral linages previously reported in other invertebrates, such as Chaq virus and several Partiti-like viruses. A reduction in the viral composition was observed during the colonization process. The present study contributes to the understanding of the viral diversity of a medically relevant vector species in its natural setting and under confinement, and sets a baseline for further studies to assess the potential implications of these viruses in the transmission of pathogens.
\end{abstract}

Keywords: Anopheles atroparvus; small RNA; Chaq virus; Partiti-like viruses; arthropod; viruses; malaria; Spain

(C) 2020 by the authors. Licensee MDPI, Basel, Switzerland. This article is an open access article distributed under the terms and conditions of the Creative Commons Attribution (CC BY) license (http://creativecommons.org/licenses/by/4.0/). 